\title{
Bilingual Keyword Extraction and its Educational Application
}

\author{
Chung-Chi Huang \\ LTI, CMU \\ 5000 Forbes Ave. \\ Pittsburgh, PA, USA \\ u901571@gmail.com
}

\author{
Mei-Hua Chen \\ FLL \\ Tunghai University \\ Taichung, Taiwan \\ chen.meihua@gmail.com
}

\author{
Ping-Che Yang \\ Institute for \\ Information Industry \\ Taipei, Taiwan
}

\begin{abstract}
We introduce a method that extracts keywords in a language with the help of the other. The method involves estimating preferences for topical keywords and fusing language-specific word statistics. At run-time, we transform parallel articles into word graphs, build crosslingual edges for word statistics integration, and exploit PageRank with word keyness information for keyword extraction. We apply our method to keyword analysis and language learning. Evaluation shows that keyword extraction benefits from cross-language information and language learners benefit from our keywords in reading comprehension test.
\end{abstract}

\section{Introduction}

Keyword extraction algorithms (KEA) have been developed to extract keywords for content understanding, event tracking, or opinion mining. However, most of them calculate article-level word keyness in $a$ single language. The articles' counterparts in another language may have different keyword candidates in mind since languages differ in grammar, phrase structure, and word usage, all of which play a role in word keyness statistics, thus keyword analysis.

Consider the English article in Figure 1. Monolingual KEA, based solely on the English content, may not identify the best keyword set. A better set might be obtained by consulting the article in more than a language (e.g., the Chinese counterparts) in that language divergence such as phrasal structure (i.e., word order), and word usage and word repetition (resulting from word translation or word sense) lead to different views on keywords across languages. Example EnglishChinese divergence in Figure 1 includes the word order in the phrase social reintegration and 重返社會 (social translated to 社會 and reintegration inversely to 重返), many-to-one mapping/translation e.g. both prosthesis and artificial limbs translated to 義肢, and one-tomany mapping e.g. physical respectively translated to 物理 and 身體 in context physical therapist and physical rehabilitation. We hypothesize that, with the differences in languages, language-specific word statistics might be fused to contribute to keyword analysis.

We present a system, BiKEA, that learns to identify keywords in a language with the help of the other. The cross-language information is expected to reinforce language similarities and respect language dissimilarities, and better understand articles in terms of keywords. An example keyword analysis of an English article is shown in Figure 1. BiKEA has aligned the parallel articles at word level and determined the topical keyword preference scores for words. BiKEA learns these topic-related scores during training by analyzing a collection of articles.

At run-time, BiKEA transforms an article in a language into PageRank word graph. To hear another side of the story, BiKEA also constructs word graph from its counterpart in another language. These two graphs are then bridged over bilingually equivalent nodes. The bridging is to take language divergence into account and 
The English Article: I've been in Afghanistan for 21 years. I work for the Red Cross and I'm a physical therapist. My job is to make arms and legs -- well it's not completely true. We do more than that. We provide the patients, the Afghan disabled, first with the physical rehabilitation then with the social reintegration. It's a very logical plan, but it was not always like this. For many years, we were just providing them with artificial limbs. It took quite many years for ...

Its Chinese Counterpart: 我在阿富汗已經 21 年。我為紅十字會工作，我是一名物理治療師。我的工 作是製作胳膊和腿--恩, 這不完全是事實。我們做的還不止這些。我們提供給患者, 阿富汗的殘疾 人, 首先是身體康復, 然後重返社會。這是一個非常合理的計劃, 但它並不是總是這樣。多年來, 我 們只是給他們提供義肢。花了很多年的程序才讓這計劃成為現在的模樣。…

Word Alignment Information: physical (物理), therapist (治療師), social (社會), reintegration (重返), physical (身體), rehabilitation (康復), prosthesis (義肢), ...

Scores of Topical Keyword Preferences for Words:

(English) prosthesis: 0.32 ; artificial leg: 0.21 ; physical therapist: 0.15 ; rehabilitation: $0.08 ; \ldots$

(Chinese) 義肢: 0.41 ; 物理治療師: 0.15 ; 康復: 0.10 ; 阿富汗: $0.08, \ldots$

English Keywords from Bilingual Perspectives:

prosthesis, artificial, leg, rehabilitation, orthopedic, ...

Figure 1. An example BiKEA keyword analysis for an English article.

to allow for language-wise interaction over word statistics. At last, BiKEA iterates in bilingual context with word keyness scores to find keywords.

\section{Related Work}

Keyword extraction has been actively applied to many NLP tasks: document categorization (Manning and Schutze, 2000), indexing (Li et al., 2004), and text mining on social networking services ((Li et al., 2010); (Zhao et al., 2011); (Wu et al., 2010)).

The body of KEA focuses on learning word statistics in document collection. Approaches such as tfidf and entropy, using local document and/or across-document information, pose strong baselines (Liu et al. (2009) and Gebre et al. (2013)). On the other hand, Mihalcea and Tarau (2004) apply PageRank, connecting words locally, to extract essential words. In our work, we integrate globally learned keyword preferences into PageRank to identify keywords.

Recent work has been incorporating semantics into PageRank. For example, Liu et al. (2010) construct PageRank synonym graph to accommodate words with similar meaning. And Huang and $\mathrm{Ku}$ (2013) weigh PageRank edges based on nodes' degrees of reference. In contrast, we bridge PageRank word graphs from parallel articles to facilitate re-distribution or interaction of the word statistics of the involved languages.

In studies more closely related to our work, Liu et al. (2010) and Zhao et al. (2011) present PageRank algorithms leveraging article topic information for keyword identification. The main differences from our current work are that the article topics we exploit are specified by humans, not automated systems, and that our PageRank graphs are built and connected bilingually.

In contrast to the previous research on topic modeling (e.g., Zhao and Xing (2007)) and keyword extraction, we present a keyword extraction algorithm that learns topical keyword preferences and bilingually inter-connects PageRank graphs. The bilinguality is to help predict better keywords taking into account the perspectives of the languages involved including the language similarities and dissimilarities. We also use our keywords for educational purpose like reading comprehension.

\section{BiKEA}

\subsection{Problem Statement}

We focus on identifying keywords of a given article in a language with the help of the other. Keyword candidates are returned as the output of the system. The returned keyword list can be examined by humans (e.g., for keyword evaluation or language learning), or passed on to article recommendation systems for article retrieval. Therefore, our goal is to return a reasonable-sized set of keyword candidates that contain the given article's essential terms. We now formally describe the problem that we are addressing.

Problem Statement: We are given a bilingual parallel article collection of various topics from social media (e.g., TED), an article $A R T^{\mathrm{e}}$ in language $e$, and its counterpart $A R T^{\mathrm{c}}$ in language $c$. Our goal is to determine a set of words that are likely to contain important words of $A R T^{\mathrm{e}}$. For 
this, we take into account word keyness w.r.t. $A R T^{e}$ 's topic and bridge language-specific statistics of $A R T^{\mathrm{e}}$ and $A R T^{\mathrm{c}}$ via bilingual information (e.g., word alignments) such that cross-lingual diversities are valued in extracting keywords in $e$.

\subsection{Topical Keyword Preferences}

We attempt to estimate language-wise keyword preferences with respect to a wide range of article topics. Basically, the estimation is to calculate word significance in a domain topic. Our learning process has following four stages.

In the first two stages of the learning process, we generate two sets of article and word information. The input to these stages is a set of articles and their domain topics. The output is a set of pairs of article ID and word in the article, e.g., (ID_ART $T^{\mathrm{e}}=1, w^{\mathrm{e}}=$ prosthesis $)$ in language $e$ or (ID_ART $T^{\mathrm{c}}=1, w^{\mathrm{c}}=$ 義肢) in language $c$, and a set of pairs of article topic and word in the article, e.g., ( $t p^{\mathrm{e}}=$ disability, $w^{\mathrm{e}}=$ prosthesis) in $e$ and $\left(t p^{\mathrm{e}}=\right.$ disability, $w^{\mathrm{c}}=$ 義肢 $)$ in $c$. Note that the topic information is shared across languages, and that, to respect language diversities, words' topical significance is calculated within their specific language and the original language-independent word statistics will later be fused and interact at run-time.

The third stage estimates keyword preferences for words across articles and domain topics using aforementioned $(A R T, w)$ and $(t p, w)$ sets. In our paper, simple yet effective tfidf estimation is used: $\quad t f i d f(w)=f r e q(A R T, w) / \operatorname{appr}(A R T,, w) \quad$ where term frequency in an article is divided by its appearance in the article collection to distinguish important words from common words.

tfidf takes global information (i.e., article collection) into account, and will be used as keyword preference model in PageRank at runtime which locally connects words (i.e., within articles).

\subsection{Run-Time Keyword Extraction}

Once language-specific keyword preference scores for words are learned, they are stored for run-time reference. BiKEA then uses the procedure in Figure 2 to fuse word statistics across languages to determine keyword list for a given article. In this procedure machine translation technique i.e., IBM word aligner is exploited to glue statistics in the involved languages and make bilingually motivated random-walk algorithm (i.e., PageRank) possible.

procedure PredictKW(ART ${ }^{\mathrm{e}}, A R T^{\mathrm{c}}$, KeyPrefs, $\left.W A, \alpha, N\right)$

//Construct language-specific word graph for PageRank

(1) $\mathbf{E W}^{\mathrm{e}}=$ constructPRwordGraph $\left(A R T^{\mathrm{e}}\right)$

(2) $\mathbf{E W}^{\mathrm{c}}=$ constructPRwordGraph $\left(A R T^{\mathrm{c}}\right)$

$/ /$ Construct inter-language bridges

(3) $\mathbf{E W}=\alpha \times \mathbf{E W}^{\mathrm{e}}+(1-\alpha) \times \mathbf{E W}^{\mathrm{c}}$ for each word alignment $\left(w_{\mathrm{i}}^{\mathrm{c}}, w_{\mathrm{j}}^{\mathrm{e}}\right)$ in $W A$ if IsContWord $\left(w_{i}{ }^{\mathrm{c}}\right)$ and IsContWord $\left(w_{\mathrm{j}}^{\mathrm{e}}\right)$

(4a) $\quad \mathbf{E W}[i, j]+=1 \times$ BiWeight $^{\text {cont }}$ else

(4b) $\quad \mathbf{E W}[i, j]+=1 \times$ BiWeight $^{\text {noncont }}$

(5) normalize each row of $\mathbf{E W}$ to sum to 1

//Iterate for PageRank

(6) set $\mathbf{K} \mathbf{P}_{1 \times v}$ to $\left[\operatorname{KeyPrefs}\left(w_{1}\right), \operatorname{KeyPrefs}\left(w_{2}\right), \ldots, \operatorname{KeyPrefs}\left(w_{\mathrm{v}}\right)\right]$

(7) initialize $\mathbf{K N}_{1 \times v}$ to $[1 / v, 1 / v, \ldots, 1 / v]$ repeat

(8a) $\mathbf{K N}^{\prime}=\lambda \times \mathbf{K N} \times \mathbf{E W}+(1-\lambda) \times \mathbf{K P}$

(8b) normalize $\mathbf{K N}$ ' to sum to 1

(8c) update $\mathbf{K N}$ with $\mathbf{K N}$ ' after the check of $\mathbf{K N}$ and $\mathbf{K N}$ ' until maxIter or avgDifference( $\left.\mathbf{K N}, \mathbf{K N} \mathbf{N}^{\prime}\right) \leq$ smallDiff

(9) rankedKeywords=Sort words in decreasing order of $\mathbf{K N}$ return the $N$ rankedKeywords in $e$ with highest scores

Figure 2. Extracting keywords at run-time.

procedure constructPRwordGraph $(A R T)$

(1) $\mathbf{E} \mathbf{W}_{v \times v}=0_{v \times v}$ for each sentence st in ART for each word $w_{\mathrm{i}}$ in $s t$ for each word $w_{\mathrm{j}}$ in st where $i<j$ and $j-i \leq W S$ if not IsContWord $\left(w_{\mathrm{i}}\right)$ and IsContWord $\left(w_{\mathrm{j}}\right)$

(2a) $\quad \mathbf{E W}[i, j]+=1 \times m$
elif not IsContWord $\left(w_{\mathrm{i}}\right)$ and not $\operatorname{IsContWord}\left(w_{\mathrm{j}}\right)$

(2b) $\quad \mathbf{E W}[i, j]+=1 \times(1 / m)$

elif IsContWord $\left(w_{\mathrm{i}}\right)$ and not IsContWord $\left(w_{\mathrm{j}}\right)$

(2c) $\quad \mathbf{E W}[i, j]+=1 \times(1 / m)$

elif IsContWord $\left(w_{\mathrm{i}}\right)$ and $\operatorname{IsContWord}\left(w_{\mathrm{j}}\right)$

(2d) $\quad \mathbf{E W}[i, j]+=1 \times m$ return $\mathbf{E W}$

Figure 3. Constructing PageRank word graph.

In Steps (1) and (2) of Figure 2 we construct PageRank word graphs for the article $A R T^{\mathrm{e}}$ in language $e$ and its counterpart $A R T^{\mathrm{c}}$ in language $c$. They are built independently using the procedure in Figure 3 to respect language properties (such as subject-verb-object or subject-object-verb structure). In the algorithm of Figure 3, EW stores normalized edge weights for word $w_{\mathrm{i}}$ and $w_{\mathrm{j}}$ (Step (2)). And EW is a $v$ by $v$ matrix where $v$ is the vocabulary size of $A R T^{\mathrm{e}}$ and $A R T^{\mathrm{c}}$. Note that the graph is directed (from words to words that follow) and edge weights are words' co-occurrences within window size WS. Additionally we incorporate edge weight multiplier $m>1$ to propagate more PageRank scores to content words. 
Then, Step (3) in Figure 2 linearly combines word graphs $\mathbf{E W}^{\mathrm{e}}$ and $\mathbf{E} \mathbf{W}^{\mathrm{c}}$ using $\alpha$. We use $\alpha$ to balance language properties/statistics, and BiKEA backs off to monolingual KEA if $\alpha$ is one.

In Step (4) for each word alignment $\left(w_{i}^{\mathrm{c}}, w_{\mathrm{j}}^{\mathrm{e}}\right)$, we construct a link between the word nodes with the weight BiWeight. The inter-language link is expected to reinforce language similarities and respect language divergence while the weight is to facilitate cross-language statistics interaction. Word alignments WA are derived using IBM models 1-5 (Och and Ney, 2003). Based on the directional word-aligned entry $\left(w_{\mathrm{i}}^{\mathrm{c}}, w_{\mathrm{j}}^{\mathrm{e}}\right)$, the inter-language link is directed from $w_{\mathrm{i}}^{\mathrm{c}}$ to $w_{\mathrm{j}}^{\mathrm{e}}$, i.e. from language $c$ to $e$. The fusion or bridging of PageRank graphs across languages is expected to help keyword extraction in language $e$ with the statistics in language $c$. Although alternative approach can be used for bridging, our approach is intuitive, and most importantly in compliance with the directional spirit of PageRank.

Step (6) sets keyword preference model KP using topical preference scores from Section 3.2, while Step (7) initializes KN of PageRank scores or, in our case, word keyness scores. Then we distribute keyness scores until KN converges. In each iteration, a word's keyness score is the linear combination of its keyword preference score and the sum of the propagation of its inbound words' previous PageRank scores. For the word $w_{\mathrm{j}}^{\mathrm{e}}$ in $A R T^{\mathrm{e}}$, any edge $\left(w_{\mathrm{i}}^{\mathrm{e}}, w_{\mathrm{j}}^{\mathrm{e}}\right)$ in $A R T^{\mathrm{e}}$, and any edge $\left(w_{\mathrm{k}}{ }^{\mathrm{c}}, w_{\mathrm{j}}^{\mathrm{e}}\right)$ in $W A$, its new PageRank score is computed as

$$
\begin{aligned}
\mathbf{K N}^{\prime}[1, j]= & \lambda \times\left(\begin{array}{c}
\alpha \times \sum_{i \in v} \mathbf{K N}[1, i] \times \mathbf{E W}^{\mathrm{e}}[i, j]+ \\
(1-\alpha) \times \sum_{k \in v} \mathbf{K N}[1, k] \times \mathbf{E W}[k, j]
\end{array}\right) \\
& +(1-\lambda) \times \mathbf{K P}[1, j]
\end{aligned}
$$

Once the iterative process stops, we rank words according to their final keyness scores and return $N$ top-ranked words in language $e$ as keyword candidates of the given article $A R T^{\mathrm{e}}$.

\section{Experiments}

\subsection{Data Sets}

We collected 3.8M-word English transcripts along with their Chinese counterparts from TED for our experiments. GENIA tagger (Tsuruoka and Tsujii, 2005) was used to lemmatize and part-of-speech tag the English transcripts while
CKIP (Ma and Chen, 2003) was used to segment the Chinese.

Fifty parallel articles (approximately 2,500 words per article) were randomly chosen and manually annotated with English keywords for keyword analysis.

\subsection{Evaluation on Keywords}

Table 1 summarizes the keyword extraction results of the baseline tfidf and our best systems on the test set. The evaluation metrics are precision, mean reciprocal rank, and $\mathrm{nDCG}$ (Jarvelin and Kekalainen, 2002).

As we can see, monolingual PageRank $(P R)$ and bilingual PageRank (BiKEA), using global information tfidf, outperform tfidf. They relatively boost $\mathrm{nDCG}$ by $21 \%$ and $\mathrm{P}$ by $55 \%$. MRR's also indicate their superiority: their toptwo candidates are often keywords vs. the $2^{\text {nd }}$ ranked from $t f i d f$. Encouragingly, BiKEA+tfidf achieves better performance than the strong monolingual $P R+t f i d f$, further improving $\mathrm{nDCG}$ relatively by $7.4 \%$ and MRR relatively by $9.4 \%$.

Overall, topical keyword preferences and inter-language bridging in PageRank which values language properties/statistics, help keyword extraction.

\begin{tabular}{|c|c|c|c|}
\hline$@ N=7$ & $\mathrm{P}$ & MRR & $\mathrm{nDCG}$ \\
\hline tfidf & .211 & .550 & .587 \\
\hline$P R+t f i d f$ & .337 & .669 & .720 \\
\hline$B i K E A+t f i d f$ & .348 & .728 & .770 \\
\hline$@ N=10$ & $\mathrm{P}$ & MRR & $\mathrm{nDCG}$ \\
\hline$\overline{t \text { tfidf }}$ & .162 & I.555 & I.594 \\
\hline$P R+t f i d f$ & .282 & .669 & .719 \\
\hline$B i K E A+t f i d f$ & .302 & .730 & .760 \\
\hline
\end{tabular}

\begin{tabular}{|l|l|l|l|}
\hline$@ N=5$ & P & MRR & nDCG \\
\hline \hline$t$ fidf & .256 & .547 & .587 \\
\hline$P R+t f i d f$ & .396 & .663 & .712 \\
BiKEA+tfidf & .412 & .725 & .765 \\
\hline
\end{tabular}

Table 1. System performance across $N$ 's.

\subsection{Application to Language Learning}

The role of highlighting keywords in reading comprehension has been attracting interest in the field of language learning and educational psychology (Nist and Hogrebe, 1987; Peterson 1991; Silvers and Kreiner, 1997). In this paper, we further examine keywords in the context of computer assisted language learning. Specifically, we applied our automatic BiKEA to keyword highlighting in reading comprehension and intended to see how much language learners can benefit from BiKEA keywords in reading comprehension test. 
This is really a two-hour presentation I give to high school students, cut down to three minutes. And it all started one day on a plane, on my way to TED, seven years ago. And in the seat next to me was a high school student, a teenager, and she came from a really poor family. And she wanted to make something of her life, and she asked me a simple little question. She said, "What leads to success?" And I felt really badly, because I couldn't give her a good answer. So I get off the plane, and I come to TED. And I think, jeez, I'm in the middle of a room of successful people! So why don't I ask them what helped them succeed, and pass it on to kids?

So here we are, seven years, 500 interviews later, and I'm gonna tell you what really leads to success and makes TEDsters tick. And the first thing is passion. Freeman Thomas says, "I'm driven by my passion." TEDsters do it for love; they don't do it for money.

Carol Coletta says, "I would pay someone to do what I do." And the interesting thing is: if you do it for love, the money comes anyway.

Work! Rupert Murdoch said to me, "It's all hard work. Nothing comes easily. But I have a lot of fun." Did he say fun? Rupert? Yes!

TEDsters do have fun working. And they work hard. I figured, they're not workaholics. They're workafrolics.

Good! Alex Garden says, "To be successful put your nose down in something and get damn good at it." There's no magic; it's practice, practice, practice.

And it's focus. Norman Jewison said to me, "I think it all has to do with focusing yourself on one thing."

And push! David Gallo says, "Push yourself. Physically, mentally, you've gotta push, push, push." You gotta push through shyness and self-doubt.

Goldie Hawn says, "I always had self-doubts. I wasn't good enough; I wasn't smart enough. I didn't think I'd make it."

Now it's not always easy to push yourself, and that's why they invented mothers. (Laughter) Frank Gehry -- Frank Gehry said to me, "My mother pushed me."

Serve! Sherwin Nuland says, "It was a privilege to serve as a doctor."

Now a lot of kids tell me they want to be millionaires. And the first thing I say to them is: "OK, well you can't serve yourself; you gotta serve others something of value. Because that's the way people really get rich."

Ideas! TEDster Bill Gates says, "I had an idea: founding the first micro-computer software company." I'd say it was a pretty good idea. And there's no magic to creativity in coming up with ideas -- it's just doing some very simple things. And I give lots of evidence.

Persist! Joe Kraus says, "Persistence is the number one reason for our success." You gotta persist through failure. You gotta persist through crap! Which of course means "Criticism, Rejection, Assholes and Pressure." (Laughter)

So, the big -- the answer to this question is simple: Pay 4,000 bucks and come to TED. Or failing that, do the eight things -- and trust me, these are the big eight things that lead to success. Thank you TEDsters for all your interviews!

Figure 4. The English TED transcript used in our reading comprehension test.

In our case study, we asked an English professor to set a multiple-choice reading comprehension test based on one English TED transcript (See Figure 4) and recruited 26 second-year college students learning English as a second language. Their proficiency in English was estimated to be of pre-intermediate level.

These students were randomly and evenly divided into experimental (reading the English transcript with BiKEA keywords) and control group (reading without). Promisingly, our keywords helped the students: students in the experimental group achieved better averaged test score (.82) than those in the control group (.74). Relatively, the improvement was $10 \%$. Moreover, post-study survey indicated that $90 \%$ of the participants found our keywords helpful for their article reading and key concept grasping. We are analyzing the influence of the highlighted BiKEA keywords on the high-performing students as well as the low-performing students in the test.

\section{Summary}

We have introduced a method for extracting keywords in bilingual context. The method involves automatically estimating topical keyword preferences and bridging languagespecific PageRank word statistics. Evaluation shows that the method can yield better keywords than strong monolingual KEA. And a case study indicates that language learners benefit from our keywords in reading comprehension test. Admittedly, using our keywords for educational purposes needs further experiments.

\section{Acknowledgement}

This study is conducted under the "Online and Offline integrated Smart Commerce Platform (2/4)" of the Institute for Information Industry which is subsidized by the Ministry of Economic Affairs of the Republic of China.

\section{References}

B. G. Gebre, M. Zampieri, P. Wittenburg, and T. Heskens. 2013. Improving native language identification with $\mathrm{tf}$-idf weighting. In Proceedings of 
the NAACL Workshop on Innovative Use of NLP for Building Educational Applications, pages 216-223.

Scott A. Golder and Bernardo A. Huberman. 2006. Usage patterns of collaborative tagging systems. Information Science, 32(2): 198-208.

Harry Halpin, Valentin Robu, and Hana Shepherd. 2007. The complex dynamics of collaborative tagging. In Proceedings of the WWW, pages 211-220.

Chung-chi Huang and Lun-wei Ku. 2013. Interest analysis using semantic PageRank and social interaction content. In Proceedings of the ICDM Workshop on Sentiment Elicitation from Natural Text for Information Retrieval and Extraction, pages 929936.

Kalervo Jarvelin and Jaana Kekalainen. 2002. Cumulated gain-based evaluation of IR technologies. ACM Transactions on Information Systems, 20(4): 422-446.

Philipp Koehn, Franz Josef Och, and Daniel Marcu. 2003. Statistical phrase-based translation. In Proceedings of the North American Chapter of the Association for Computational Linguistics, pages 4854.

Quanzhi Li, Yi-Fang Wu, Razvan Bot, and Xin Chen. 2004. Incorporating document keyphrases in search results. In Proceedings of the Americas Conference on Information Systems.

Zhenhui Li, Ging Zhou, Yun-Fang Juan, and Jiawei Han. 2010. Keyword extraction for social snippets. In Proceedings of the $W W W$, pages 1143-1144.

Marina Litvak and Mark Last. 2008. Graph-based keyword extraction for single-document summarization. In Proceedings of the ACL Workshop on Multi-Source Multilingual Information Extraction and Summarization, pages 17-24.

F. Liu, D. Pennell, F. Liu, and Y. Liu. 2009. Unsupervised approaches for automatic keyword extraction using meeting transcripts. In Proceedings of the NAACL, pages 620-628.

Zhengyang Liu, Jianyi Liu, Wenbin Yao, and Cong Wang. 2010. Keyword extraction using PageRank on synonym networks. In Proceedings of the ICEEE, pages 1-4.

Zhiyuan Liu, Wenyi Huang, Yabin Zheng, and Maosong Sun. 2010. Automatic keyphrase extraction via topic decomposition. In Proceedings of the EMNLP, pages 366-376.

Wei-Yun Ma and Keh-Jiann Chen. 2003. Introduction to CKIP Chinese word segmentation system for the first international Chinese word segmentation bakeoff. In Proceedings of the ACL Workshop on Chinese Language Processing.

Chris D. Manning and Hinrich Schutze. 2000. Foundations of statistical natural language processing. MIT Press.

Rada Mihalcea and Paul Tarau. 2004. TextRank: Bringing orders into texts. In Proceedings of the EMNLP, pages 404-411.

S. L. Nist and M. C. Hogrebe. 1987. The role of underlining and annotating in remembering textual information. Reading Research and Instruction, 27(1): $12-25$.

Franz Josef Och and Hermann Ney. 2003. A systematic comparison of various statistical alignment models. Computational Linguistics, 29(1): 19-51.

Divya Padmanabhan, Prasanna Desikan, Jaideep Srivastava, and Kashif Riaz. 2005. WICER: a weighted inter-cluster edge ranking for clustered graphs. In Proceedings of the IEEE/WIC/ACM WI, pages 522-528.

S. E. Peterson. 1991. The cognitive functions of underlining as a study technique. Reading Research and Instruction, 31(2): 49-56.

V. L. Silvers and D. S. Kreiner. 1997. The effects of preexisting inappropriate highlighting on reading comprehension. Reading Research and Instruction, 36(3): 217-223.

Yoshimasa Tsuruoka and Jun'ichi Tsujii. 2005. Bidirectional inference with the easiest-first strategy for tagging sequence data. In Proceedings of the EMNLP, pages 467-474.

Peter D. Turney. 2000. Learning algorithms for keyphrase extraction. Information Retrieval, 2(4): 303-336.

Wei Wu, Bin Zhang, and Mari Ostendorf. 2010. Automatic generation of personalized annotation tags for Twitter users. In Proceedings of the NAACL, pages 689-692.

B. Zhao and E. P. Xing. 2007. HM-BiTAM: Bilingual topic exploration, word alignment, and translation. In Proceedings of the NIPS.

Wayne Xin Zhao, Jing Jiang, Jing He, Yang Song, Palakorn Achananuparp, Ee-Peng Lim, and Xiaoming Li. 2011. Topical keyword extraction from Twitter. In Proceedings of the ACL, pages 379-388. 